\title{
Analysis of vibroprotection characteristics of pneumatic relaxation seat suspension with capability of vibration energy recuperation
}

\author{
Mikhail Lyashenko, Pavel Potapov ${ }^{1}$, and Azamat Iskaliev $^{1, *}$ \\ ${ }^{1}$ Volgograd State Technical University (VSTU), department «Transport vehicles and engines», 400005, Volgograd, Lenin Ave 28, \\ Russia
}

\begin{abstract}
This paper proposes mechanism and control algorithm for pneumatic relaxation system of suspension with vibration energy recuperation applied to standard vehicle operator seat ("Sibeko" company). Mathematical model of the seat pneumatic relaxation suspension with two additional air volumes was created. Pneumatic motor - recuperator activated by means of air flow from the one additional volume to another is installed in air piping between additional volumes. Computational research was made in Matlab/Simulink. Amplitude-frequency characteristics of transmission coefficient for standard and proposed suspensions were plotted for preliminary evaluation of vibration protection properties of seat suspension. Performed comparative analysis of amplitude-frequency characteristics shows that noticeable improvement of vibration protection properties of pneumatic relaxation suspension system with vibration energy recuperation in comparison with standard system both in region of resonance disturbances and in aboveresonance region. Main ways for further improvement of vibration protection properties of proposed system were marked out.
\end{abstract}

At the modern stage of development of ground vehicles high values of operational characteristics (for example, specific power and operational speeds) led to the problem of improvement of the functional characteristics of suspension systems. These systems must provide necessary riding comfort during performing of technological operations and effective protection of operator from unhealthy influence of the low-frequency vibrations and noise.

At the present time, operator's seat suspensions with nonlinear characteristics are believed to be the most effective. For example, suspensions with the pneumatic elastic element are widely used in modern tractors $[6,7]$. Standard unregulated shock absorber installed in these suspensions provides necessary damping of vibrations in the resonance zone but makes worse protection from vibrations in the above- resonance zone [2]. There are ways with various efficiency for solving of this problem, for example, using of active suspensions $[3,8]$. But the results of the performed analysis show that the most preferable one is the self-regulated suspension system with the semi-active control that doesn't require external power sources and operate effective in the whole frequency diapason of impacts. Also, availability of function of recuperation of absorbed vibration energy clearly increases efficiency factor and efficiency of the suspension in comparison with other suspensions that have standard unregulated dampers which dissipate vibration energy. This paper presents the proposed controllable pneumatic relaxation suspension of seat with the feature of recuperation of vibration energy. This suspension was created on base of the driver seat made by "Sibeko" company.

The research was aimed at determination of ways for improvement of protection from vibration for the onesupport suspension system by means of using of the pneumatic relaxation suspension with availability of vibration energy recuperation. Object of research was widely using in automotive technique "Sibeko" seat [5] that has pneumatic suspension with automatic regulation of static position.

In results of the stand experiments static elasticdamping characteristic of the standard seat suspension was obtained [1]. Analysis of the characteristic shows that significant area of hysteresis curve indicates a presence in the suspension mechanism (mainly, in hydraulic shock absorber) of dry friction forces with high values. These forces negatively affects on seat protection from vibrations with high-frequency excitations [2]. In accordance with the proposed concept of control of suspension stiffness, energy absorption and its further recuperation must takes place on areas at the end of compression and rebound strokes. At these stages relative velocity of suspension mass changes sign and potential energy reaches extreme values. The authors propose scheme and the method of control of the onesupport suspension which allow implementation of the relaxation suspension that meet requirements of proposed concept (Fig. 


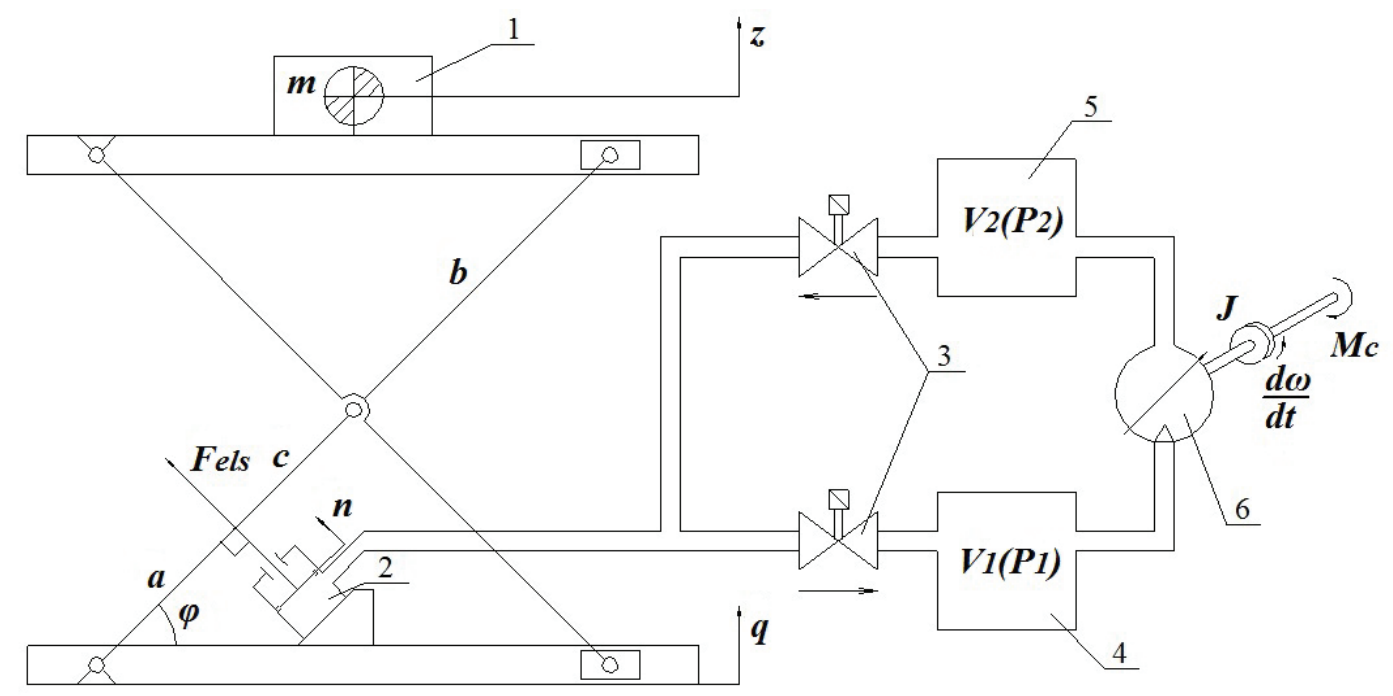

Fig. 1. Design scheme of the pneumatic suspension with two additional volumes: 1 - sprung body with mass m; 2 - displacement of elastic element; 3 - controlled electric valves; 4,5 - additional volumes; 6 - pneumatic motor

Absorption of vibration energy is performed by means of operation of valves 3 . Opening of valves 3 is performed in the next manner: the first valve allows flow of a gas mass from the volume 2 to the first volume 4 at the end of compression stroke and the second valve from the volume 5 to the volume 2 at the end of rebound stroke. Pneumatic motor (recuperator of energy) installed between additional volumes performs effective work by means of variable difference of air pressure in mentioned volumes.

The mathematical model was created in accordance with the main statements of theoretical mechanic and gas dynamic.

Equation of dynamic of the sprung body with mass m:

$$
m \cdot \ddot{z}=F_{e l s} \cdot \cos \varphi-F_{n}-F_{f},
$$

where $\ddot{z}$ - current value of acceleration of sprung body, $\mathrm{m} / \mathrm{sec}^{2} ; F_{\text {els }}$ - elastic force, $N ; \varphi$ - current value of the tilt angle between lever of directing mechanism and base, $\mathrm{rad} ; F_{n}=m \cdot g\left(g=9,81 \mathrm{~m} / \mathrm{sec}^{2}\right)-$ gravity force, $N$; $F_{f}-$ friction force, $N$.

Elastic force:

$$
F_{e l s}=\left(P_{p}-P_{a}\right) \cdot S_{p},
$$

where $P_{p}$ - current value of air pressure in working chamber, $\mathrm{Pa} ; \mathrm{P}_{a}$ - atmosphere pressure, $\mathrm{Pa} ; S_{p}$ - area of piston in the elastic element, $m^{2}$.

Current value of the tilt angle between lever of directing mechanism and base:

$$
\varphi=\arcsin \left(\frac{z-q}{(a+b+c)}+\sin \varphi_{0}\right)
$$

where $z, q$ - current values of displacements of base and sprung body, $m$; $a, b, c$ - components of length of lever of direction mechanism, $m ; \varphi_{0}$ - initial value of the tilt angle between lever of directing mechanism and base, rad.

Friction force:

$$
F_{f}=T_{0} \cdot \operatorname{sign}(\dot{z}-\dot{q})
$$

where $T_{0}, N$, (value was defined in experiment [1]); $\dot{z}, \dot{q}$ - current velocities of sprung body and base, $\mathrm{m} / \mathrm{sec}$.

Kinematic harmonic impact on base:

$$
q=A_{0} \cdot \sin (\omega \cdot t),
$$

where $A_{0}=0,03 \mathrm{~m}$ - amplitude of excitation; $\omega-$ frequency of excitation, $\mathrm{sec}^{-1} ; t$ - time, sec.

Speed of change of air pressure in working chamber:

$$
\dot{P}_{p}=\frac{\chi \cdot P_{p} \cdot \dot{m}_{2 p}}{m_{p}}-\frac{\chi \cdot P_{p} \cdot \dot{m}_{p 1}}{m_{p}}-\frac{\chi \cdot P_{p} \cdot S_{p} \cdot \dot{n}}{V_{p}},
$$

where $\chi$-polytrophic index; $m_{p}$ - current mass of air in working chamber, $\mathrm{kg} ; \dot{m}_{2 p}, \dot{m}_{p 1}$ - current air flow rate from the second additional volume to working chamber and from working chamber to the first additional volume respectively, $\mathrm{kg} / \mathrm{sec} ; \dot{n}$ - current velocity of piston rod in working chamber of elastic element, $\mathrm{m} / \mathrm{sec} ; V_{p}-$ current volume of air in working chamber, $\mathrm{m}^{3}$.

Current volume of air in working chamber:

$$
V_{p}=V_{p 0}+S_{p} \cdot n
$$

where $V_{p 0}$ - initial static volume of air in working chamber, $m^{3} ; n$ - current coordinate of piston rod position in working chamber of elastic element, $m$.

Current coordinate of piston rod position in working chamber of elastic element: 


$$
n=a \cdot\left(\operatorname{tg} \varphi-\operatorname{tg} \varphi_{0}\right)
$$

Speed of change of air pressure in the first additional volume:

$$
\dot{P}_{1}=\frac{\chi \cdot P_{1} \cdot \dot{m}_{p 1}}{m_{1}}-\frac{\chi \cdot P_{1} \cdot \dot{m}_{12}}{m_{1}},
$$

where $P_{1}-$ current air pressure in the first additional volume, $\mathrm{Pa} ; \mathrm{m}_{1}$ - current air mass in the first additional volume, $\mathrm{kg} ; \dot{m}_{12}$ - current air flow rate from the first additional volume, $\mathrm{kg} / \mathrm{sec}$.

Speed of change of air pressure in the second additional value:

$$
\dot{P}_{2}=\frac{\chi \cdot P_{2} \cdot \dot{m}_{12}}{m_{2}}-\frac{\chi \cdot P_{2} \cdot \dot{m}_{2 p}}{m_{2}},
$$

where $P_{2}$ - current air pressure in the second additional value, $\mathrm{Pa} ; \mathrm{m}_{2}$ - current air mass in the second additional volume, $\mathrm{kg}$. body:

Air pressure in system at static position of sprung

$$
P_{p 0}=P_{10}=P_{20}=\frac{F_{\mathrm{g}}}{\cos \varphi_{0} \cdot S_{p}}+P_{a}
$$

where $P_{p 0}, P_{10}, P_{20}$ - air pressure in working chamber and in additional volumes at static position of sprung body, $P a$. body:

Air density in system at static position of sprung

$$
\rho_{p 0}=\rho_{10}=\rho_{20}=\rho_{a} \cdot\left(\frac{P_{p 0}}{P_{a}}\right)
$$

where $\rho_{p 0}, \rho_{10}, \rho_{20}$ - air density in working chamber and in additional volumes at static position of sprung body, $\mathrm{kg} / \mathrm{m}^{3} ; \rho_{a}-$ air density at atmosphere pressure, $\mathrm{kg} / \mathrm{m}^{3}$.

Current air mass in working chamber:

$$
m_{p}=\rho_{p 0} \cdot V_{p} \cdot\left(\frac{P_{p}}{P_{p 0}}\right)^{\frac{1}{\chi}}
$$

Current air mass in the first additional volume:

$$
m_{1}=\rho_{10} \cdot V_{1} \cdot\left(\frac{P_{1}}{P_{10}}\right)^{\frac{1}{\chi}}
$$

where $V_{1}$ - value of the first additional volume, $m^{3}$.

Current air mass in the second additional volume:

$$
m_{2}=\rho_{20} \cdot V_{2} \cdot\left(\frac{P_{2}}{P_{20}}\right)^{\frac{1}{\chi}},
$$

where $V_{2}$-value of the second additional volume, $\mathrm{m}^{3}$.

Equation of rotational dynamic of shaft of pneumatic motor:

$$
\frac{d \omega}{d t}=\frac{\left(P_{1}-P_{2}\right) \cdot F_{\psi} \cdot r-M_{c}}{J},
$$

where $\frac{d \omega}{d t}$ - current angular acceleration of pneumatic motor shaft, $\sec ^{-2} ; F_{\psi}$ - area of influence of air positive pressure, $m^{2} ; r$ - length of arm of influence, $m ; M_{c}-$ resistance torque of pneumatic motor drive, $N^{*} m ; J-$ inertia moment of pneumatic motor shaft, $\mathrm{kg}^{*} \mathrm{~m}^{2}$.

Resistance torque of pneumatic motor drive:

$$
M_{c}=M_{c 0}+\sigma_{0} \cdot \omega^{2} \text {, }
$$

where $M_{c 0}$ - initial resistance torque ("rest"), $N^{*} m ; \sigma_{0}$ - correction coefficient; $\omega$ - angular speed of pneumatic motor shaft, $\sec ^{-1}$.

Flow section of air line:

$$
F=\frac{\pi \cdot d^{2}}{4}
$$

where $d$-diameter of air line cross-section, $m$.

Flow of working body from one volume to another could be assumed adiabatic if flow sections are sufficiently small.

Current air flow rate from working chamber to the first additional volume:

$$
\frac{P_{1}}{P_{p}}>0,528
$$

$\dot{m}_{p 1}=\mu \cdot F \cdot \sqrt{\frac{2 \cdot k}{k-1} \cdot \frac{P_{p} \cdot m_{p}}{V_{p}} \cdot\left[\left(\frac{P_{1}}{P_{p}}\right)^{\frac{2}{k}}-\left(\frac{P_{1}}{P_{p}}\right)^{\frac{k+1}{k}}\right]}$;

when:

$$
\frac{P_{1}}{P_{p}} \leq 0,528
$$

$$
\dot{m}_{p 1}=\mu \cdot F \cdot \sqrt{\frac{2 \cdot k}{k-1} \cdot \frac{P_{p} \cdot m_{p}}{V_{p}} \cdot\left[\left(\frac{2}{k+1}\right)^{\frac{2}{k-1}}-\left(\frac{2}{k+1}\right)^{\frac{k+1}{k-1}}\right]},
$$

where $\mu$ - coefficient of air flow through controlled valve; $k$ - adiabatic index.

Current air flow rate from the first additional volume to the second additional volume:

when

$$
\begin{gathered}
\left(P_{1}-P_{2}\right) \cdot F_{\psi} \cdot r-M_{c}>0, \\
\frac{P_{2}}{P_{1}}>0,528
\end{gathered}
$$




$$
\dot{m}_{12}=\mu^{*} \cdot F_{\psi} \cdot \sqrt{\frac{2 \cdot k}{k-1} \cdot \frac{P_{1} \cdot m_{1}}{V_{1}} \cdot\left[\left(\frac{P_{2}}{P_{1}}\right)^{\frac{2}{k}}-\left(\frac{P_{2}}{P_{1}}\right)^{\frac{k+1}{k}}\right]} \text {; }
$$

when

$$
\begin{gathered}
\left(P_{1}-P_{2}\right) \cdot F_{\psi} \cdot r-M_{c}>0, \\
\frac{P_{2}}{P_{1}} \leq 0,528
\end{gathered}
$$

$$
\dot{m}_{12}=\mu^{*} \cdot F_{\psi} \cdot \sqrt{\frac{2 \cdot k}{k-1} \cdot \frac{P_{1} \cdot m_{1}}{V_{1}} \cdot\left[\left(\frac{2}{k+1}\right)^{\frac{2}{k-1}}-\left(\frac{2}{k+1}\right)^{\frac{k+1}{k-1}}\right]},
$$

where $\mu^{*}-$ coefficient of air flow in cavity of pneumatic motor drive.

Current air flow rate from the second additional volume to working chamber:

when:

$$
\frac{P_{p}}{P_{2}}>0,528
$$

$$
\dot{m}_{2 p}=\mu \cdot F \cdot \sqrt{\frac{2 \cdot k}{k-1} \cdot \frac{P_{2} \cdot m_{2}}{V_{2}} \cdot\left[\left(\frac{P_{p}}{P_{2}}\right)^{\frac{2}{k}}-\left(\frac{P_{p}}{P_{2}}\right)^{\frac{k+1}{k}}\right]}
$$

when

$$
\frac{P_{p}}{P_{2}} \leq 0,528
$$

$\dot{m}_{2 p}=\mu \cdot F \cdot \sqrt{\frac{2 \cdot k}{k-1} \cdot \frac{P_{2} \cdot m_{2}}{V_{2}} \cdot\left[\left(\frac{2}{k+1}\right)^{\frac{2}{k-1}}-\left(\frac{2}{k+1}\right)^{\frac{k+1}{k-1}}\right]}$.

Proposed algorithm of valves working is presented in table 1.

Table 1. Control algorithm of valves working

\begin{tabular}{|c|c|c|}
\hline \multicolumn{3}{|c|}{ Compression stroke } \\
\hline$\dot{m}_{p 1}>0 ; \dot{m}_{2 p}=0$ & $\dot{z}-\dot{q}<0$ & $0>z>-A_{0}$ \\
\cline { 1 - 1 }$\dot{m}_{p 1}=0 ; \dot{m}_{2 p}=0$ & & $z \leq-A_{0}$ \\
\hline$\dot{m}_{p 1}>0 ; \dot{m}_{2 p}=0$ & $\dot{z}-\dot{q}=0$ & $z<0$ \\
\hline$\dot{m}_{p 1}=0 ; \dot{m}_{2 p}>0$ & $\dot{z}-\dot{q}>0$ & $z<0$ \\
\hline \multicolumn{2}{|c|}{ Rebound stroke } \\
\hline$\dot{m}_{p 1}=0 ; \dot{m}_{2 p}>0$ & $\dot{z}-\dot{q}>0$ & $0<z<A_{0}$ \\
\hline$\dot{m}_{p 1}=0 ; \dot{m}_{2 p}=0$ & & $z \geq A_{0}$ \\
\hline$\dot{m}_{p 1}=0 ; \dot{m}_{2 p}>0$ & $\dot{z}-\dot{q}=0$ & $z>0$ \\
\hline$\dot{m}_{p 1}>0 ; \dot{m}_{2 p}=0$ & $\dot{z}-\dot{q}<0$ & $z>0$ \\
\hline
\end{tabular}

To evaluate potential characteristics of protection from vibration of the proposed suspension, it is necessary to determinate its work for absorption of vibration energy. Given that, $z-q=-\lambda$, where $\lambda-$ suspension deformation, in accordance with the elastic- damping characteristic (fig. 2) obtained with the algorithm (table 1) it is possible to define work for absorption of vibration energy and recuperator efficiency coefficient.

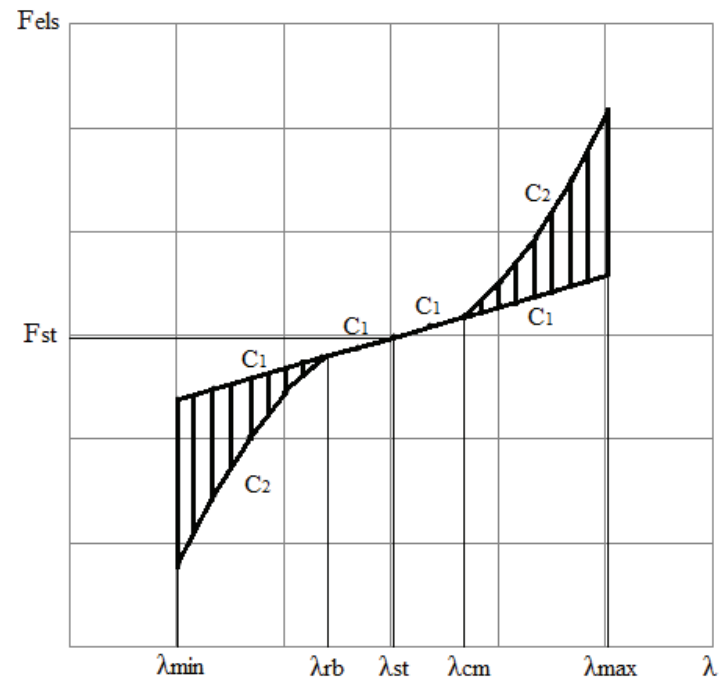

Fig. 2. Theoretical elastic-damping characteristic

Work for absorption done by the suspension during one period of vibrations (fig. 2):

$$
\begin{aligned}
& L_{a b s(t=T)}=\int_{\lambda_{c m}}^{\lambda_{\max }}\left(c_{2}-c_{1}\right) \cdot \lambda \cdot d \lambda+\int_{\lambda_{\min }}^{\lambda_{r b}}\left(\left(c_{1}+c_{2}\right) \cdot \lambda+\right. \\
& \left.+c_{1} \cdot\left(\lambda_{\min }-\lambda_{r b}\right)\right) \cdot d \lambda,
\end{aligned}
$$

where $c_{1}, c_{2}$ - suspension stiffness values at opening and closing of the controlled valves accordingly; $\lambda_{\min }, \lambda_{\max }$ - suspension deformations at the ends of rebound and compression strokes; $\lambda_{r b}, \lambda_{c m}$ - suspension deformations corresponds to the moment of closing of the controlled valves; $\lambda_{s t}$-values of suspension static deformation.

Power of the recuperator:

$$
\begin{aligned}
& \int_{0}^{N_{r e c}} d N_{r e c}=\int_{0}^{\omega} M_{c} \cdot d \omega=M_{c 0} \cdot \int_{0}^{\omega} d \omega+\sigma_{0} \cdot \int_{0}^{\omega} \omega^{2} d \omega, \\
& N_{r e c}=M_{c 0} \cdot \omega+\sigma_{0} \cdot \frac{\omega^{3}}{3} .
\end{aligned}
$$

Efficience work done by recuperator during one period of vibrations:

$$
\int_{0}^{L_{r e c}(t=T)} d L_{r e c}=\int_{0}^{T} N_{r e c} d t
$$

Efficiency coefficient of recuperator:

$$
\eta_{r e c}=\frac{L_{r e c(t=T)}}{L_{a b s(t=T)}} .
$$

Presented mathematical model was created in Simulink Matlab. Equations were solved by means of 
Dormand-Price method with variable integrator step. Example of a model part for one differential equation (9) in Simulink is presented in fig. 3 .

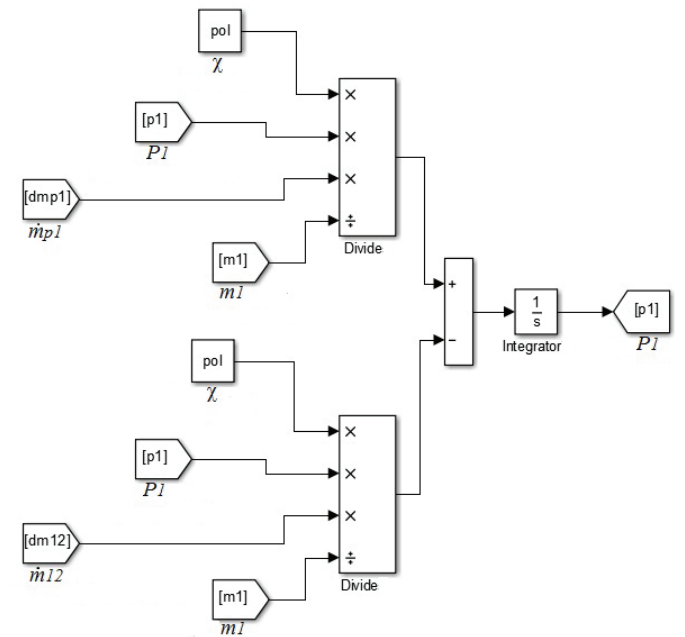

Fig. 3. Example of a model part for one differential equation (9) in Simulink

Graphs of amplitude-frequency characteristics of transfer coefficients (fig. 4) for standard and proposed suspensions (fig. 2) were plotted to initially evaluate characteristics of protection from vibrations.

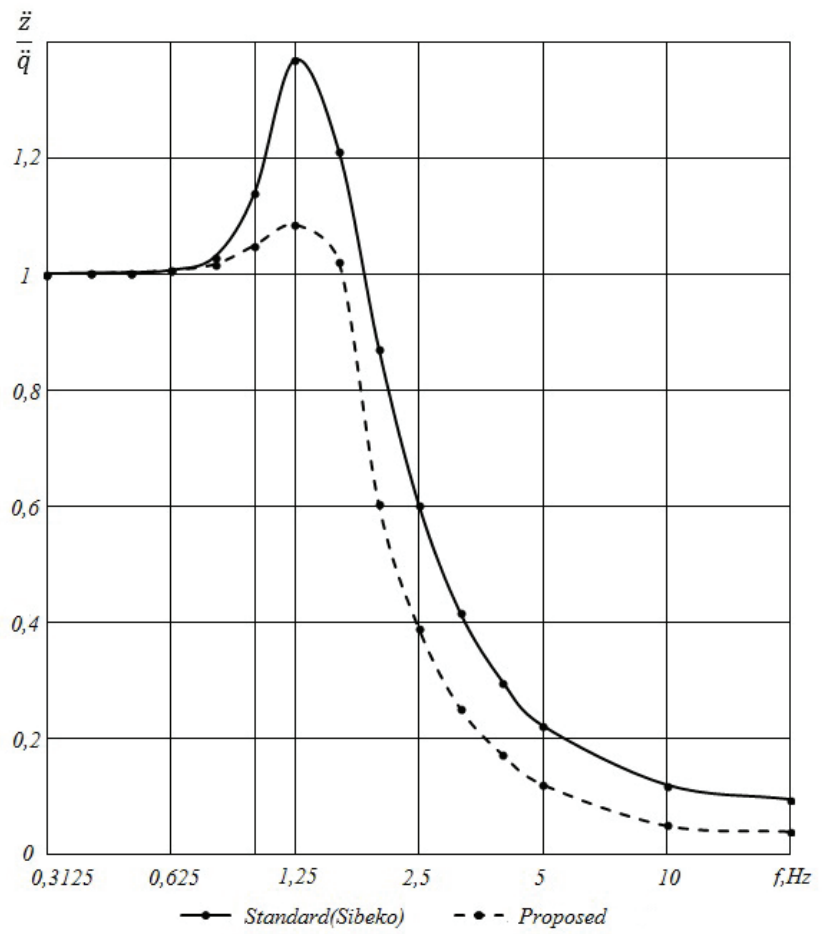

Fig. 4. Amplitude-frequency characteristics of transfer coefficients

Results analysis shows that the proposed suspension system has significantly better protection from vibrations both in resonance zone, and in high-frequency zone in comparison with the standard "Sibeko" suspension. Values of transfer coefficient of the proposed suspension system in resonance zone of impacts are 1.19 times less and in over-resonance zone 2 times in comparison with transfer coefficient of the standard suspension.

Results of presented research show that the proposed mechanism and control algorithm for the pneumatic relaxation system with vibration energy recuperation could be efficiency used for both one-support, and multisupport suspension systems of ground vehicles. Further improvement of proposed suspension systems would be performed by means of design search of possibilities for increasing of value of absorbed vibration energy. It allows significantly decrease hydraulic damping or even fully reject standard hydraulic shock absorber in prospective.

\section{References}

1. A. Iskaliev, M. Ljashenko, Proceedings of IV international scientific and practical conference "Scientific and technical progress: topical and prospective directions of future", 181-183 (2016)

2. V. Novikov, I. Ryabov, K.V. Chernyshov, Vibroprotection characteristics of automotive suspensions (RPK Politekhnik, Volgograd, 2009)

3. O. Polivaev, A. Yushin, O. Kostikov, Tractors and agricultural machinery, 9, 47-49, 2008

4. A. Pozdeev, Truck, 6, 2-6, 2011

5. http://sibeko-russia.ru/catalog/8/27/1253.html.

6. http://www.claas.ru/produkte/traktoren.

7. http://www.deutz-fahr.com/ru-RU/products/tractors.

8. A. Yushin, Improvement of using efficiency of tractor-transport aggregate by means of modernization of suspension of tractor seat: abstract of thesis for candidate of technical science degree (Voronezh, 2007) 